\title{
A Min, + System Theory for Constrained Traffic Regulation and Dynamic Service Guarantees
}

\author{
Cheng-Shang Chang, Senior Member, IEEE, Rene L. Cruz, Senior Member, IEEE, Jean-Yves Le Boudec, and \\ Patrick Thiran, Member, IEEE
}

\begin{abstract}
By extending the system theory under the (min, +) algebra to the time-varying setting, we solve the problem of constrained traffic regulation and develop a calculus for dynamic service guarantees. For a constrained traffic-regulation problem with maximum tolerable delay $d$ and maximum buffer size $q$, the optimal regulator that generates the output traffic conforming to a subadditive envelope $f$ and minimizes the number of discarded packets is a concatenation of the $g$-clipper with $g(t)=\min [f(t+$ $d), f(t)+q]$ and the maximal $f$-regulator. The $g$-clipper is a bufferless device, which optimally drops packets as necessary in order that its output be conformant to an envelope $g$. The maximal $f$-regulator is a buffered device that delays packets as necessary in order that its output be conformant to an envelope $f$. The maximal $f$-regulator is a linear time-invariant filter with impulse response $f$, under the $(\min ,+)$ algebra.

To provide dynamic service guarantees in a network, we develop the concept of a dynamic server as a basic network element. Dynamic servers can be joined by concatenation, "filter bank summation," and feedback to form a composite dynamic server. We also show that dynamic service guarantees for multiple input streams sharing a work-conserving link can be achieved by a dynamic service curve earliest deadline scheduling algorithm, if an appropriate admission control is enforced.
\end{abstract}

Index Terms-Buffer overflow, (min, +) algebra, network calculus, packet losses, performance analysis, traffic shaping.

\section{INTRODUCTION}

$\mathbf{F}$ UTURE high-speed digital networks aim to provide integrated services, including voice, video, fax, and data. To control interaction among traffic generated by different sources, traffic regulation seems inevitable. In [10], Cruz proposed the following deterministic traffic characterization. A traffic stream, described by a nondecreasing sequence

Manuscript received October 15, 2001; revised January 30, 2002; approved by IEEE/ACM TRANSACTIONS ON NETWORKING Editor M. Ammar. This work was supported in part by the National Science Council, Taiwan, R.O.C., under Contract NSC-87-2213-E007-084 and Contract NSC-88-2213-E007-004, by the U.S. National Science Foundation under Grant NCR 94-15684 and Grant NCR 97-25568, by the Center for Wireless Communications at the University of California at San Diego, and by the Priority Program in Information and Communication Structures of the Swiss National Science under Grant SPP ICS 5003-45311.

C.-S. Chang is with the Department of Electrical Engineering, National Tsing Hua University, Hsinchu, 30043 Taiwan, R.O.C. (e-mail: cschang@ee.nthu.edu.tw).

R. L. Cruz is with the Department of Electrical and Computer Engineering, University of California at San Diego, La Jolla, CA 92093-0407 USA (e-mail: cruz@ece.ucsd.edu).

J.-Y. Le Boudec and P. Thiran are with the School of Computer and Communication Sciences, Ecole Polytechnique Fédérale de Lausanne, Luasanne CH-1015, Switerland (e-mail: jean-yves.leboudec@epfl.ch).

Digital Object Identifier 10.1109/TNET.2002.804824
$A \equiv\{A(t), t=0,1,2$,$\} [with A(0)=0$ ], conforms to a function $f$, called an envelope, if

$$
A(t)-A(s) \leq f(t-s) \quad \forall s \leq t .
$$

Without loss of generality, an envelope $f$ can be assumed to be subadditive [6], i.e., $f(s)+f(t-s) \geq f(t)$ for all $s \leq t$. Using this characterization, a calculus is developed in [10] and [11] to compute deterministic performance measures, such as bounds on delay and bounds on queue length. Traffic regulation addresses the problem of modifying a traffic stream so that it conforms to a subadditive envelope $f$. The problem of traffic regulation was treated systematically in [8] and [20], where it is shown that the optimal traffic regulator that generates an output $B$ conforming to a subadditive envelope $f$ for an input $A$ is a linear time-invariant filter with the impulse response $f$ under the $(\min ,+)$ algebra, i.e.,

$$
B(t)=\min _{0 \leq s \leq t}[A(s)+f(t-s)] .
$$

We call such a filter the maximal $f$-regulator. This characterization was also observed in [1], [2], and [27].

As the buffer in the maximal $f$-regulator is assumed to be infinite, packets from the input might be queued at the regulator. For a real-time service, the delay of a queued packet at the regulator might exceed a maximum tolerable delay and such a packet should be discarded (i.e., clipped). The problem of traffic regulation with such a delay constraint is called the constrained traffic-regulation problem in [19]. Its objective is to find a regulator that not only generates traffic conforming to an envelope, but also minimizes the number of discarded packets. In addition to the delay constraint, Konstantopoulos and Anantharam [19] also considered the buffer constraint for the regulator. For $f(t)=\rho t+\sigma$, they derived optimal traffic regulators that satisfied either the delay constraint or the buffer constraint.

Cruz and Taneja [16] considered the zero delay case of the constrained traffic-regulation problem. This is also the case without any buffer. By extending the time-invariant filtering theory under the $(\mathrm{min},+)$ algebra to the time-varying setting, it is shown there that the departure process of the optimal zero-delay regulator, which generates a departure process conformant to $f$, is the subadditive closure [8] of the arrival process convolved with $f$. Such a bufferless regulator is called the $f$-clipper in [16].

Motivated by all these works, one of the main objectives of this paper is to provide an optimal and implementable solution for the general constrained traffic-regulation problem with both 
the delay and buffer constraints. As in [16], our approach is based on the time-varying filtering theory under the $(\min ,+)$ algebra. By extending the subadditive closure in [8] to the timevarying setting, we show that the $f$-clipper with input $A$ and output $B$ can be implemented using the following recursive equation:

$$
\begin{aligned}
B(t)=\min [B(t-1)+A(t)-A(t-1), & \\
& \left.\min _{0 \leq s<t}[B(s)+f(t-s)]\right] .
\end{aligned}
$$

The computation complexity of the $f$-clipper is almost the same as that of the maximal $f$-regulator. The recursive equation also implies that the $f$-clipper is greedy. Packets are discarded only when needed.

For the constrained traffic-regulation problem with maximum tolerable delay $d$ and maximum buffer size $q$, the optimal traffic regulator is shown to be a concatenation of the $g$-clipper with $g(t)=\min [f(t)+q, f(t+d)]$ and the maximal $f$-regulator. The solution is intuitive as the output from the $g$-clipper conforms to the envelope $g$ that yields bounded delay $d$ and bounded queue length $q$ at the maximal $f$-regulator. For example, when $f(t)=$ $\min _{1 \leq i<K}\left[\rho_{i} t+\sigma_{i}\right]$, the corresponding $g$-clipper can be implemented by $K$ parallel bufferless $\left(\sigma_{i}+\min \left[q, \rho_{i} d\right], \rho_{i}\right)$-leaky buckets. A packet is discarded if it cannot be admitted to one of these $K$ leaky buckets. The output from the $g$-clipper is then fed into $K$ parallel $\left(\sigma_{i}, \rho_{i}\right)$-leaky buckets.

In addition, the time-varying filtering theory can also be used for dynamic service guarantees. By extending the concept of the service curve in [1], [12], and [20] to a bivariate function $F(\cdot, \cdot)$, we define a dynamic $F$-server for an input $A$ if its output $B$ satisfies

$$
B(t) \geq \min _{0 \leq s \leq t}[A(s)+F(s, t)] \quad \forall t .
$$

Analogous to the time-invariant filtering theory in [1], [8], and [20], a dynamic $F$-server can be viewed as a linear filter with the time-varying impulse response $F$. It can be combined by concatenation, "filter bank summation," and feedback to form a composite dynamic server. We illustrate the use of the dynamic server by considering a work-conserving link with a timevarying capacity and a dynamic window-flow-control problem. We also show that dynamic service guarantees for multiple input streams sharing a work-conserving link can be achieved by a dynamic service curve earliest deadline (SCED) scheduling algorithm if an appropriate admission control is enforced. As the SCED algorithm in [27], the dynamic SCED algorithm is an earliest deadline first (EDF) policy that schedules packets according to their deadlines.

The remainder of this paper is organized as follows. In Section II, we introduce the time-varying filtering theory under the $(\mathrm{min},+)$ algebra. The development is parallel to the time-invariant filtering theory in [1], [8], and [20]. The reader is also referred to [3] and [4], which contain results overlapping with this paper. In Sections III and IV, we introduce the maximal dynamic traffic regulators and maximal dynamic clippers, respectively. These are used for solving the problem of constrained traffic regulation in Section V. In Section VI, we develop the concept of dynamic servers and their associated calculus. We show in Section VII that the dynamic SCED algorithm can be used to achieve dynamic service guarantees. We conclude the paper in Section VIII by discussing possible extensions and applications.

\section{Time-VARYing Filtering Theory Under THE Min, + ALGEBRA}

In the section, we introduce the time-varying filtering theory under the $(\min ,+)$ algebra. The development is parallel to the time-invariant filtering theory in [1], [8], and [20]. To extend the $(\min ,+)$ algebra to the time-varying setting, we consider the family of bivariate functions

$\tilde{\mathcal{F}}=\{F(\cdot, \cdot): F(s, t) \geq 0, F(s, t) \leq F(s, t+1)$, for all $0 \leq s \leq t\}$.

Thus, for any $F \in \tilde{\mathcal{F}}, F(s, t)$ is nonnegative and nondecreasing in $t$. For any two bivariate functions $F$ and $G$ in $\tilde{\mathcal{F}}$, we say $F=$ $G$ (respectively, $F \leq G$ ) if $F(s, t)=G(s, t)$ [respectively, $F(s, t) \leq G(s, t)]$ for all $0 \leq s \leq t$. We define the following two operations for functions in $\tilde{\mathcal{F}}$.

i) (min) the pointwise minimum of two functions

$$
(F \oplus G)(s, t)=\min [F(s, t), G(s, t)] .
$$

ii) (convolution) the convolution of two functions under the $(\mathrm{min},+)$ algebra

$$
(F \star G)(s, t)=\min _{s \leq \tau \leq t}[F(s, \tau)+G(\tau, t)] .
$$

One can easily verify that $(\tilde{\mathcal{F}}, \oplus, \star)$ is a complete dioid (see, e.g., [5]) with the zero function $\tilde{\epsilon}$ and the identity function $\tilde{e}$, where $\tilde{\epsilon}(s, t)=\infty$ for all $s \leq t$, and $\tilde{\mathbf{e}}(s, t)=0$ if $s=t$ and $\infty$ otherwise. To be precise, we have the following properties.

1. (Associativity) $\forall F, G, H \in \tilde{\mathcal{F}}$

$$
\begin{aligned}
(F \oplus G) \oplus H & =F \oplus(G \oplus H) \\
(F \star G) \star H & =F \star(G \star H) .
\end{aligned}
$$

2. (Commutativity) $\forall F, G \in \tilde{\mathcal{F}}$

$$
F \oplus G=G \oplus F .
$$

3. (Distributivity for infinite "sums") For any two sequences of functions $F_{m}$ and $G_{m}$ in $\tilde{\mathcal{F}}$

$$
\left(\bigoplus_{i=1}^{\infty} F_{i}\right) \star\left(\bigoplus_{j=1}^{\infty} G_{j}\right)=\bigoplus_{i=1}^{\infty} \bigoplus_{j=1}^{\infty}\left(F_{i} \star G_{j}\right) .
$$

4. (Zero element) $\forall F \in \tilde{\mathcal{F}}$

$$
F \oplus \tilde{\epsilon}=F .
$$

5. (Absorbing zero element) $\forall F \in \tilde{\mathcal{F}}$

$$
F \star \tilde{\epsilon}=\tilde{\epsilon} \star F=\tilde{\epsilon} .
$$

6. (Identity element) $\forall F \in \tilde{\mathcal{F}}$

$$
F \star \tilde{\mathbf{e}}=\tilde{\mathbf{e}} \star F=F .
$$


7. (Idempotency of addition) $\forall F \in \tilde{\mathcal{F}}$

$$
F \oplus F=F \text {. }
$$

The key difference to the time-invariant filtering theory is that we do not have the commutative property for $\star$ in $(\tilde{\mathcal{F}}, \oplus, \star)$, i.e., $F \star G \neq G \star H$ in general.

Let $\tilde{\mathcal{F}}_{0}=\{F \in \tilde{\mathcal{F}}: F \oplus \tilde{\mathbf{e}}=F\}$. That is, a function $F \in \tilde{\mathcal{F}}_{0}$ if $F(t, t)=0$ for all $t$. As in the time-invariant case, we still have the following monotonicity.

8. (Monotonicity) $\forall F \leq \tilde{F}, G \leq \tilde{G}$

$$
\begin{aligned}
& F \oplus G \leq \tilde{F} \oplus \tilde{G} \leq \tilde{F} \\
& F \star G \leq \tilde{F} \star \tilde{G} .
\end{aligned}
$$

If $F$ (respectively, $G$ ) is in $\tilde{\mathcal{F}}_{0}$, then $F \star G \leq G$ (respectively, $F \star G \leq F$ ). If both $F$ and $G$ are in $\tilde{\mathcal{F}}_{0}$, then $F \oplus G \geq F \star G$.

For any function $F \in \tilde{\mathcal{F}}$, define the unitary operator (called the closure operation in this paper)

$$
\begin{aligned}
F^{*} & =\lim _{n \rightarrow \infty}(F \oplus \tilde{\mathbf{e}})^{(n)} \\
& =\lim _{n \rightarrow \infty}\left(\tilde{\mathbf{e}} \oplus F \oplus F^{(2)} \oplus \cdots \oplus F^{(n)}\right)
\end{aligned}
$$

where $F^{(n)}$ is the self-convolution of $F$ for $n$ times, i.e., $F^{(n)}=$ $F^{(n-1)} \star F, n \geq 2$, and $F^{(1)}=F$. The limit in (1) exists as ( $\left.\tilde{\mathbf{e}} \oplus F \oplus F^{(2)} \oplus \cdots \oplus F^{(n)}\right)$ is decreasing in $n$. Expanding (1) yields

$$
F^{*}(s, t)=\inf _{S} \sum_{i=1}^{m}\left[F\left(t_{i-1}, t_{i}\right)\right]
$$

where $S=\left\{t_{0}, t_{1}, t_{2}, \ldots, t_{m}\right\}$ is any subset of $\{1,2, \ldots, t\}$ with $t_{0}=s<t_{1}<t_{2}<\cdots<t_{m}=t$.

In addition to the algebraic properties, we present several important properties in Lemmas 2.1 and 2.2 that will be used to prove results for constrained traffic regulation and service guarantees.

Lemma 2.1: Suppose that $F, G \in \tilde{\mathcal{F}}$.

i) (Monotonicity) If $F \leq G$, then $F^{*} \leq G^{*}$.

ii) (Closure properties) $F^{*}=F^{*} \oplus \tilde{\mathbf{e}}=F^{*} \star F^{*}=$ $\left(F^{*}\right)^{(m)}=\left(F^{*}\right)^{*} \leq F \oplus \tilde{\mathbf{e}} \leq F$.

iii) (Maximum solution) $F^{*}$ is the maximum solution of the equation $H=(H \star F) \oplus \tilde{\mathbf{e}}$, i.e., for any $H$ satisfying $H=(H \star F) \oplus \tilde{\mathbf{e}}, H \leq F^{*}$.

iv) $F^{*}$ can be computed recursively from the following equations:

$$
\begin{aligned}
& F^{*}(s, s)=0 \\
& F^{*}(s, t)=\min _{s \leq \tau<t}\left[F^{*}(s, \tau)+F(\tau, t)\right] .
\end{aligned}
$$

v) $(F \oplus G)^{*}=\left(F^{*} \oplus G^{*}\right)^{*}=\left(F^{*} \star G^{*}\right)^{*}$.

Proof: As the proofs for i)-iv) are identical to those in [8] and [9], we only prove v). From the monotonicity, $F \oplus G \geq$ $F^{*} \oplus G^{*} \geq F^{*} \star G^{*}$. Thus, $(F \oplus G)^{*} \geq\left(F^{*} \star G^{*}\right)^{*}$. On the other hand, one has $F \geq F \oplus G$. Thus, $F^{*} \geq(F \oplus G)^{*}$. Similarly, $G^{*} \geq(F \oplus G)^{*}$. This implies

$$
F^{*} \star G^{*} \geq(F \oplus G)^{*} \star(F \oplus G)^{*}=(F \oplus G)^{*} .
$$

Thus,

$$
\left(F^{*} \star G^{*}\right)^{*} \geq\left((F \oplus G)^{*}\right)^{*}=(F \oplus G)^{*} .
$$

Lemma 2.2 (Feedback): Suppose that $F, G, H \in \tilde{\mathcal{F}}$.

i) For the equation

$$
H=(H \star F) \oplus G
$$

$H=G \star F^{*}$ is the maximum solution.

ii) If $\inf _{t} F(t, t)>0$, then $H=G \star F^{*}$ is the unique solution.

iii) Under the condition in ii), if

$$
H \geq(H \star F) \oplus G
$$

then $H \geq G \star F^{*}$.

The proofs for Lemma 2.2 are identical to those in [8] and [9] and, thus, are omitted.

Remark 2.3: As in [8], let $\mathcal{F}=\{f: f(0) \geq 0, f(s) \leq f(t)$, $s \leq t\}$ be the set of nonnegative and nondecreasing functions. Also, let $\mathcal{F}_{0}$ be the subset of functions in $\mathcal{F}$ with $f(0)=0$. One may then define the convolution of a function $f \in \mathcal{F}$ and a bivariate function $G \in \tilde{\mathcal{F}}$ as follows:

$$
(f \star G)(t)=\min _{0 \leq s \leq t}[f(s)+G(s, t)] .
$$

Under such a definition, $f \star G$ is in $\mathcal{F}$. One may view $f \star G$ as a special case of $F \star G$ for some $F \in \tilde{\mathcal{F}}$ with $F(0, t)=f(t)$ for all $t$ and $F(s, t)=\infty$, for all $t$ and $s>0$. Thus, the results in Lemma 2.2 still hold.

Remark 2.4: A bivariate function $F$ is time invariant if

$$
F(s, t)=F(s+u, t+u) \quad \forall s \leq t \text { and } u \geq 0 .
$$

By letting $f(t)=F(0, t)$, one can easily verify that $F$ is time invariant if and only if there exists some $f \in \mathcal{F}$ such that $F(s, t)=f(t-s)$. As a result, time-invariant bivariate functions commute. To see this, consider two invariant functions $F$ and $G$ and let $f(t)=F(0, t)$ and $g(t)=G(0, t)$. Then

$(F \star G)(s, t)=\min _{s \leq u \leq t}[f(u-s)+g(t-u)]=(G \star F)(s, t)$.

An important corollary of (4) is that Lemma 2.1(v) can be simplified as follows (cf. [8, Lemma 2.2(xi)]):

$$
(F \oplus G)^{*}=\left(F^{*} \oplus G^{*}\right)^{*}=\left(F^{*} \star G^{*}\right)^{*}=F^{*} \star G^{*} .
$$

Remark 2.5: A bivariate function $F$ is additive if

$$
F(s, u)+F(u, t)=F(s, t) \quad \forall s \leq u \leq t .
$$

For an additive bivariate function $F$, one easily check that

$$
F^{(2)}(s, t)=\min _{s \leq u \leq t}[F(s, u)+F(u, t)]=F(s, t)
$$

which implies that $F^{*}=F$. Note that a bivariate function $F$ is additive if and only if there is a function $f \in \mathcal{F}$ such that $F(s, t)=f(t)-f(s)$. This can be easily verified by choosing $f(t)=F(0, t)$. 


\section{DyNAMIC TRAFFIC REgULATION}

Given a sequence $A \in \mathcal{F}_{0}$, it is defined in [10] and [11] that $A$ conforms to the (static) upper envelope $f \in \mathcal{F}_{0}$ if $A(t)-A(s) \leq$ $f(t-s)$ for all $s \leq t$. It is also shown in [8] and [1] that the optimal traffic regulator that generates output traffic conforming to a subadditive envelope $f$ is a linear time-invariant filter with the impulse response $f$ under the $(\mathrm{min},+)$ algebra. In this section, we extend such a result to the time-varying setting.

We start from extending the definition of a static envelope to a dynamic envelope.

Definition 3.1: A sequence $A \in \mathcal{F}_{0}$ is said to conform to the dynamic upper envelope $F \in \tilde{\mathcal{F}}_{0}$ if for all $s \leq t$ there holds $A(t)-A(s) \leq F(s, t)$.

As in [8] and [9], this characterization has the following equivalent statements. The proof is omitted.

Lemma 3.2: Suppose that $A \in \mathcal{F}_{0}$ and $F \in \tilde{\mathcal{F}}_{0}$. The following statements are equivalent.

i) $A$ conforms to the dynamic upper envelope $F$.

ii) $A=A \star F$.

iii) $A=A \star F^{*}$.

iv) $A$ conforms to the dynamic upper envelope $F^{*}$.

Given a dynamic upper envelope $F \in \tilde{\mathcal{F}}_{0}$, one can construct a regulator such that, for any input $A \in \mathcal{F}_{0}$, the output from the regulator conforms to the dynamic upper envelope $F$. This is done in the following theorem. Once again, the proof is omitted.

Theorem 3.3: Suppose that $A \in \mathcal{F}_{0}$ and $F \in \tilde{\mathcal{F}}_{0}$. Let $B=$ $A \star F^{*}$.

i) (Traffic regulation) $B$ conforms to the dynamic upper envelope $F^{*}$ and, thus, $B$ also conforms to the dynamic upper envelope $F$.

ii) (Flow constraint) $B \leq A$.

iii) (Optimality) For any $\tilde{B} \in \mathcal{F}_{0}$ that satisfies i) and ii), one has $\tilde{B} \leq B$.

iv) (Conformity) $A$ conforms to the dynamic upper envelope $F$ if and only if $B=A$.

The construction $B=A \star F^{*}$ is called the maximal dynamic $F$-regulator (for the input $A$ ).

As in the time-invariant case, the flow constraint $B \leq A$ corresponds to one of the causal conditions in [19] as the number of departures cannot be larger than the number of arrivals. Theorem 3.3(iii) shows that, under the flow constraint and the constraint that the output traffic conforms to the dynamic upper envelope $F$, the maximal $F$-regulator is the best construction that one can implement.

Example 3.4 (Work-Conserving Link With a Time-Varying Capacity): Consider a work-conserving link with a time-varying capacity. Let $c(t)$ be the maximum number of packets that can be served at time $t, C(t)=\sum_{\tau=1}^{t} c(\tau)$ be the cumulative capacity in the interval $[1, t]$, and $\hat{C}(s, t)=C(t)-C(s)$ be the cumulative capacity in the interval $[s+1, t]$. Let $A(t)$ and $B(t)$ be the input and the output from the work-conserving link. Denote by $q(t)$ the number of packets at the link at time $t$. The work-conserving link is then governed by Lindley's equation

$$
q(t+1)=[q(t)+A(t+1)-A(t)-c(t+1)]^{+}
$$

where $x^{+}=\max [0, x]$. Suppose $q(0)=0$. Recursive expansion of Lindley's equation yields

$$
q(t)=\max _{0 \leq s \leq t}[A(t)-A(s)-\hat{C}(s, t)] .
$$

Since $q(t)=A(t)-B(t)$, we have

$$
B(t)=\min _{0 \leq s \leq t}[A(s)+\hat{C}(s, t)] .
$$

As $\hat{C}$ is an additive bivariate function, we have from Remark 2.5 that $\hat{C}^{*}=\hat{C}$, which shows that the work-conserving link is the maximal dynamic $\hat{C}$-regulator. This example also shows that the calculation of the convolution in (8) can be easily implemented by the recursion in (6).

We note that a work-conserving link with a time-varying capacity is also equivalent to a time-varying (greedy) shaper in [20].

Example 3.5 (Traffic Regulation With a Capacity Constraint): Consider a link with a time-varying capacity. The link is not necessarily work conserving. As in the previous example, let $c(t)$ be the maximum number of packets that can be served at time $t$, and $C(t)=\sum_{\tau=1}^{t} c(\tau)$ be the cumulative capacity by time $t$. Let $A(t)$ and $B(t)$ be the input and output from the link. Though the link may not be work conserving, the output $B$ is still constrained by the capacity, i.e.,

$$
B(t)-B(s) \leq C(t)-C(s) .
$$

Suppose that we would like to perform traffic regulation for the input $A$ such that the output $B$ conforms to the static envelope $f \in \mathcal{F}$, i.e.,

$$
B(t)-B(s) \leq f(t-s) \quad \forall s \leq t .
$$

From Theorem 3.3, we know that the optimal implementation for the output to satisfy (9) and (10) is the maximal dynamic $F$-regulator with

$$
F(s, t)=\min [C(t)-C(s), f(t-s)] .
$$

If $c(t)$ is bounded above by $c_{\max }>0$ and if the cumulative time-varying capacity $C$ is bounded below by some curve $h \in$ $\mathcal{F}$ over any time window, i.e., if for all $0 \leq s \leq t, h(t-s) \leq$ $C(t)-C(s) \leq c_{\max }(t-s)$, then one can derive static service curves bounding below the maximal dynamic $F$-regulator (11). Such curves are obtained in [15], [22], and [23].

\section{DyNAMIC TRAFFIC CliPPING}

The maximal dynamic $F$-regulator solves the traffic-regulation problem with an infinite buffer. In this section, we consider the traffic-regulation problem without a buffer. The question is then how one drops packets optimally such that the output conforms to a dynamic envelope $F$. Such a problem was previously solved in [16]; however, the solution in [16] cannot be easily implemented directly. In the following theorem, we present a recursive construction for the solution.

Theorem 4.1: Suppose that $A \in \mathcal{F}_{0}$ and $F \in \tilde{\mathcal{F}}_{0}$. Let $B(t)=$ $(\hat{A} \oplus F)^{*}(0, t)$, where $\hat{A}(s, t)=A(t)-A(s)$. The following statements then hold. 
i) (Traffic regulation) $B$ conforms to the dynamic upper envelope $F$.

ii) (Clipping constraint) $B(t)-B(t-1) \leq A(t)-A(t-1)$ for all $t$.

iii) (Optimality) For any $\tilde{B} \in \mathcal{F}_{0}$ that satisfies i) and ii), one has $\tilde{B} \leq B$.

iv) $B$ can be constructed by the following recursive equation:

$$
\begin{aligned}
B(t)=\min [B(t-1)+A(t) & -A(t-1), \\
& \left.\min _{0 \leq s<t}[B(s)+F(s, t)]\right]
\end{aligned}
$$

with $B(0)=0$.

v) (Conformity) $A$ conforms to the dynamic upper envelope $F$ if and only if $B=A$.

The construction in (12) is called the maximal dynamic $F$-clipper (for the input $A$ ) in this paper.

Proof: For any $s \leq t$, we have from Lemma 2.1(iv) that

$$
\begin{aligned}
B(t) & =(\hat{A} \oplus F)^{*}(0, t) \\
& \leq(\hat{A} \oplus F)^{*}(0, s)+(\hat{A} \oplus F)(s, t) \\
& \leq B(s)+F(s, t)
\end{aligned}
$$

and, hence, $B \leq B \star F$ so that $B$ is conformant to $F$, establishing i).

To see ii), note similarly that

$$
\begin{aligned}
B(t) & =(\hat{A} \oplus F)^{*}(0, t) \\
& \leq(\hat{A} \oplus F)^{*}(0, t-1)+(\hat{A} \oplus F)(t-1, t) \\
& \leq B(t-1)+\hat{A}(t-1, t) .
\end{aligned}
$$

Next, we establish iii). Suppose that $\tilde{B} \in \mathcal{F}_{0}$ satisfies i) and ii). Since $\tilde{B}(0)=0$

$$
\tilde{B} \leq \mathbf{e}
$$

where $\mathbf{e}(0)=0$ and $\mathbf{e}(s)=\infty$ for $s>0$. As $\tilde{B}$ conforms to the dynamic envelope $F$

$$
\tilde{B} \leq \tilde{B} \star F .
$$

The inequality in the clipping constraint in ii) is equivalent to $\tilde{B}(t)-\tilde{B}(s) \leq A(t)-A(s)$ for all $s \leq t$ and it can be rewritten as

$$
\tilde{B} \leq \tilde{B} \star \hat{A}
$$

with $\hat{A}(s, t)=A(t)-A(s)$. The constraints in (13)-(15) are equivalent to

$$
\tilde{B}=\tilde{B} \oplus(\tilde{B} \star F) \oplus(\tilde{B} \star \hat{A}) \oplus \mathbf{e} .
$$

Applying the distributivity and the fact that $\hat{A} \in \tilde{\mathcal{F}}_{0}$ yields

$$
\begin{aligned}
\tilde{B} & =(\tilde{B} \star(\tilde{\mathbf{e}} \oplus \hat{A} \oplus F)) \oplus \mathbf{e} \\
& =(\tilde{B} \star(\hat{A} \oplus F)) \oplus \mathbf{e} .
\end{aligned}
$$

It then follows from Lemma 2.2(i) that $\mathbf{e} \star(\hat{A} \oplus F)^{*}$ is the maximum solution of (16). Note that

$$
\left(\mathbf{e} \star(\hat{A} \oplus F)^{*}\right)(t)=(\hat{A} \oplus F)^{*}(0, t)=B(t) .
$$

Thus, $B$ is the maximum solution that satisfies i) and ii).

To see iv), note from Lemma 2.1(iv) that $B$ can be constructed recursively as follows:

$$
\begin{aligned}
B(t)= & \min _{0 \leq s<t}[B(s)+\min [A(t)-A(s), F(s, t)]] \\
= & \min \left[\min _{0 \leq s<t}[B(s)+A(t)-A(s)],\right. \\
& \left.\min _{0 \leq s<t}[B(s)+F(s, t)]\right]
\end{aligned}
$$

with $B(0)=0$. Since $B$ satisfies the clipping constraint,

$$
\begin{aligned}
B(s) & +A(t)-A(s) \\
& =B(s)+A(t-1)-A(s)+A(t)-A(t-1) \\
& \geq B(s)+B(t-1)-B(s)+A(t)-A(t-1) \\
& =B(t-1)+A(t)-A(t-1) .
\end{aligned}
$$

This implies that

$$
\min _{0 \leq s<t}[B(s)+A(t)-A(s)]=B(t-1)+A(t)-A(t-1) .
$$

Thus,

$$
\begin{aligned}
B(t)=\min [B(t-1)+A(t) & -A(t-1), \\
& \left.\min _{0 \leq s<t}[B(s)+F(s, t)]\right] .
\end{aligned}
$$

To prove v), note that if $B=A$, then it follows from (18) that $A=A \star F$. Thus, $A$ conforms to the dynamic envelope $F$. On the other hand, if $A$ conforms to the dynamic envelope $F$, then

$$
A(t)-A(s) \leq F(s, t) .
$$

This implies $\hat{A} \oplus F=\hat{A}$. As $\hat{A}^{*}=\hat{A}$ from Remark 2.5

$$
B(t)=(\hat{A} \oplus F)^{*}(0, t)=A(t)-A(0)=A(t) .
$$

We note that the original representation in [16] is that $B(t)=$ $\left(\hat{A} \star F^{*}\right)^{*}(0, t)$. This is equivalent to our result in Theorem 4.1, as can be seen from Lemma 2.1(v), and the fact that $\hat{A}^{*}=\hat{A}$. As $(\hat{A} \oplus F)^{*}=\left(\hat{A} \oplus F^{*}\right)^{*}$ in Lemma 2.1(v), one also has the following equivalent implementation:

$$
\begin{aligned}
B(t)=\min [B(t-1)+A(t) & -A(t-1), \\
& \left.\min _{0 \leq s<t}\left[B(s)+F^{*}(s, t)\right]\right] .
\end{aligned}
$$

Note that the key difference between Theorems 3.3 and 4.1 is the clipping constraint. The clipping constraint implies that, in any given slot, the packets departing are a subset of the packets arriving in the same slot. Let $\ell(t)=A(t)-A(t-1)-(B(t)-$ 
$B(t-1))$ be the number of packets clipped at time $t$. From (12), we have

$$
\begin{aligned}
\ell(t)=\max [0, B(t-1)+ & A(t)-A(t-1) \\
& -\min _{0 \leq s<t}[B(s)+F(s, t)] .
\end{aligned}
$$

Observe from (20) that packet loss occurs at time $t$ only when at least one of the following inequalities is violated:

$$
\begin{aligned}
& (B(t-1)+A(t)-A(t-1))-B(s) \\
& \quad \leq F(s, t), \quad s=0,1 \ldots, t-1 .
\end{aligned}
$$

When this happens, one then discards packets to the extent so that the above inequalities are all satisfied. Note also that (12) implies that the maximal dynamic $F$-clipper can be implemented in real time since the value of $B(t)$ depends only on $B(s-1)$ and $A(s)$ for $s \leq t$.

In the following example, we illustrate how one implements the maximal dynamic $F$-clipper by a work-conserving link with a finite buffer when $F(s, t)=\rho(t-s)+q$ for $s<t$.

Example 4.2 (Work-Conserving Link With a Finite Buffer): Consider the work-conserving link with a time-varying capacity in Example 3.4. In addition, we assume that the buffer size of the link is $q$, i.e., at most, $q$ packets can be stored at the link. Packets that arrive at the link and find the buffer full are lost. As in Example 3.4, let $A(t)$ and $B(t)$ be the input and output from the work-conserving link. Denote by $q(t)$ the number of packets at the link at time $t$.

We then need to modify Lindley's equation in (6) as follows:

$$
q(t+1)=\min \left[[q(t)+A(t+1)-A(t)-c(t+1)]^{+}, q\right] .
$$

The number of lost packets at time $t$, denoted by $\ell(t)$, is then $\max [q(t-1)+A(t)-A(t-1)-c(t)-q, 0]$. Let $A_{1}$ be the effective input to the link, i.e.,

$$
A_{1}(t)-A_{1}(t-1)=A(t)-A(t-1)-\ell(t) .
$$

For the effective input $A_{1}$, the work-conserving link behaves like a work-conserving link with an infinite buffer. Thus, we have from (7) that

$$
q(t)=\max _{0 \leq s \leq t}\left[A_{1}(t)-A_{1}(s)-\hat{C}(s, t)\right]
$$

assuming $q(0)=0$. This then implies

$$
\begin{aligned}
\ell(t)=\max [q(t-1)+A(t)-A(t-1)-c(t)-q, 0] \\
=\max \left[0, \max _{0 \leq s \leq t-1}\left[A_{1}(t-1)-A_{1}(s)-\hat{C}(s, t-1)\right]\right. \\
\quad+A(t)-A(t-1)-c(t)-q] \\
=\max \left[0, A_{1}(t-1)+A(t)-A(t-1)\right. \\
\left.\quad-\min _{0 \leq s<t}\left[A_{1}(s)+\hat{C}(s, t)+q\right]\right] .
\end{aligned}
$$

In view of (20), the effective input $A_{1}$ to the work-conserving link with a finite buffer is, in fact, the output of the maximal dynamic $F$-clipper with $F(s, t)=\hat{C}(s, t)+q, s<t$. In particular, when $c(t)=\rho$ for all $t$, we can implement the maximal dynamic $F$-clipper with $F(s, t)=\rho(t-s)+q$ by constructing the effective input of a work-conserving link with constant capacity $\rho$ and buffer $q$. This example also shows that a direct calculation of the convolution in (12) may not be necessary, and the convolution in this example can be computed recursively by (22).

For the maximal dynamic $F$-clipper with the input $A$ and the output $B$, let $L(t)=A(t)-B(t)$ be the cumulative losses at the clipper by time $t$. As $B(t)=(\hat{A} \oplus F)^{*}(0, t)$ in Theorem 4.1, using (2) yields

$$
\begin{aligned}
L(t)= & A(t)-\inf _{S} \sum_{i=1}^{m} \min \left[A\left(t_{i}\right)-A\left(t_{i-1}\right), F\left(t_{i-1}, t_{i}\right)\right] \\
= & \sup _{S}\left[A(t)-\sum_{i=1}^{m} \min \left[A\left(t_{i}\right)-A\left(t_{i-1}\right), F\left(t_{i-1}, t_{i}\right)\right]\right] \\
= & \sup _{S}\left[\sum_{i=1}^{m}\left(A\left(t_{i}\right)-A\left(t_{i-1}\right)\right)\right. \\
& \left.\quad-\sum_{i=1}^{m} \min \left[A\left(t_{i}\right)-A\left(t_{i-1}\right), F\left(t_{i-1}, t_{i}\right)\right]\right] \\
= & \sup _{S} \sum_{i=1}^{m}\left[A\left(t_{i}\right)-A\left(t_{i-1}\right)-F\left(t_{i-1}, t_{i}\right)\right]^{+}
\end{aligned}
$$

where $S=\left\{t_{0}, t_{1}, t_{2}, \ldots, t_{m}\right\}$ is any subset of $\{1,2, \ldots, t\}$ with $t_{0}=0<t_{1}<t_{2}<\cdots<t_{m}=t$. This was previously shown in [16, Corollary 1]. A similar result is also obtained in [22] for both the continuous and discrete time settings.

Example 4.3 (Clippers in Tandem): Now we compare the output from the maximal dynamic $F_{1} \oplus F_{2}$-clipper and a concatenation of the maximal dynamic $F_{1}$-clipper and the maximal dynamic $F_{2}$-clipper. Let $A$ be the input to both systems, $B_{1}$ be the output from the maximal dynamic $F_{1}$-clipper, $B_{2}$ be the output from the maximal dynamic $F_{2}$-clipper, and $B$ be the output from the maximal dynamic $F_{1} \oplus F_{2}$-clipper. Also let $L(t)=A(t)-B(t)$ be the cumulative losses at the maximal dynamic $F_{1} \oplus F_{2}$-clipper by time $t$. Similarly, let $L_{1}(t)=$ $A(t)-B_{1}(t)$ and $L_{2}(t)=B_{1}(t)-B_{2}(t)$. From Theorem 4.1, we have for all $s \leq t$

$$
\begin{aligned}
& B_{1}(t)-B_{1}(s) \leq A(t)-A(s) \\
& B_{1}(t)-B_{1}(s) \leq F_{1}(s, t) \\
& B_{2}(t)-B_{2}(s) \leq B_{1}(t)-B_{1}(s) \\
& B_{2}(t)-B_{2}(s) \leq F_{2}(s, t) .
\end{aligned}
$$

This implies that $B_{2}$ conforms to the dynamic upper envelope $F_{1} \oplus F_{2}$ and that $B_{2}(t)-B_{2}(t-1) \leq A(t)-A(t-1)$. Thus, $B_{2} \leq B$ and $L(t) \leq L_{1}(t)+L_{2}(t)$ for all $t$ by Theorem 4.1. In fact, a concatenation of the maximal dynamic $F_{1}$-clipper and maximal dynamic $F_{2}$-clipper is a suboptimal implementation of an $F_{1} \oplus F_{2}$-clipper. The reason for this, as observed in [16], is that the discarding of packets in the $F_{2}$-clipper is not accounted for in the $F_{1}$-clipper. 
Example 4.4 (Clippers in Parallel): Continue from the previous example. Since clippers in tandem are suboptimal and may yield more cumulative losses than the optimal one, we may use this to compare the cumulative losses for clippers in parallel. Now suppose both the maximal dynamic $F_{1}$-clipper and maximal dynamic $F_{2}$-clipper are fed with the input $A$. Let $B_{1}^{\prime}$ and $B_{2}^{\prime}$ be the outputs from these two clippers and $L_{1}^{\prime}(t)=$ $A(t)-B_{1}^{\prime}(t)$ and $L_{2}^{\prime}(t)=A(t)-B_{2}^{\prime}(t)$ be the cumulative losses at these two clippers by time $t$. Clearly, $L_{1}^{\prime}(t)=L_{1}(t)$. It is easy to see from (24) that $L_{2}^{\prime}(t) \geq L_{2}(t)$. Thus, we still have $L(t) \leq L_{1}^{\prime}(t)+L_{2}^{\prime}(t)$ for all $t$. This is previously reported in [16, Corollary 2].

\section{Constrained Traffic Regulation}

The two traffic-regulation problems, with an infinite buffer and without a buffer, are two extreme cases. In practice, packets (or cells) may be queued and delayed at a regulator. However, there might be constraints for the buffer size and the delay. In this regard, one might have to discard (i.e., clip) some packets from the input so that the buffer and delay constraints can be satisfied. The question is then how one discard packets optimally so that the number of clipped packets can be minimized. Such a problem is called constrained traffic regulation and was first considered in [19] for $(\sigma, \rho)$-leaky buckets. Our objective in this section is to provide a general, simple, and optimal solution for the constrained traffic-regulation problem.

To formalize the problem of constrained traffic regulation with buffer and delay constraints, we let $A$ be the input and $B$ be the output from the regulator. We require that the buffer occupancy in the regulator be less than or equal to $q$, the delay be bounded above by $d$, and that the output $B$ be conformant to a dynamic envelope $F$. Due to these constraints, packets may need to be discarded. Let $A_{1}$ be the effective input, i.e., $A_{1}(t)$ counts the total number of packets arriving up to and including slot $t$, which eventually depart the regulator without being discarded. The objective is to maximize the effective input $A_{1}$ and the output $B$, given the buffer and delay constraints and the constraint that $B$ conforms to the dynamic envelope $F$. More formally, given the input $A$ and a dynamic envelope $F$, we seek $A_{1}$ and $B$, which are as large as possible subject to the following constraints.

(C1) (Clipping constraint) $A_{1}(t)-A_{1}(t-1) \leq A(t)$ $A(t-1)$ for all $t$.

(C2) (Buffer constraint) $A_{1}(t)-q \leq B(t)$ for all $t$, where $q$ is the buffer size at the regulator.

(C3) (Delay constraint) $A_{1}(t) \leq B(t+d)$ for all $t$, where $d$ is the maximum tolerable delay at the regulator (as the regulator serves packets in the FCFS order).

(C4) (Traffic regulation) $B$ conforms to the dynamic upper envelope $F$.

(C5) (Flow constraint) $B(t) \leq A_{1}(t)$ for all $t$.

The clipping constraint implies that the packets in the effective input $A_{1}$ is a subset of the packets in $A$ for any time $t$. We note that the clipping constraint does not imply that packets arriving at time $t$ have to be clipped at time $t$. In fact, they could be clipped at some time later than $t$. However, as will be shown below, optimal clipping can be greedy and only those packets arriving at time $t$ need to be clipped at time $t$. Note also that the natural buffer constraint should be $A_{1}^{\prime}(t)-B(t) \leq q$, where $A_{1}^{\prime}(t)$ is the cumulative number of packets arriving up to time $t$, which have not been discarded at the end of slot $t$. Our buffer constraint $A_{1}(t)-B(t) \leq q$ is, in fact, less restrictive as $A_{1}(t) \leq A_{1}^{\prime}(t)$ for all $t$. However, as the below theorem shows, the optimal value of $A_{1}(t)$ can be computed without knowledge of $A(s)$ for $s>t$ so that packets that will eventually be discarded in an optimal clipper can, in fact, be discarded when they arrive. Assuming this is the case, the backlog of packets in the optimal regulator at the end of slot $t$ is $A_{1}(t)-B(t)$.

Theorem 5.1: Suppose that $A \in \mathcal{F}_{0}$ and $F \in \tilde{\mathcal{F}}_{0}$. Let $A_{1}$ be the output from the maximal dynamic $G$-clipper for the input $A$, where

$$
\begin{aligned}
& G(s, s)=0 \quad \forall s \\
& G(s, t)=\min \left[F^{*}(s, t+d), F^{*}(s, t)+q\right] \quad \forall s<t .
\end{aligned}
$$

Also, let $B$ be the output from the maximal dynamic $F$-regulator for the input $A_{1}$. All constraints (C1)-(C5) are then satisfied. Moreover, for any $\tilde{A}_{1}, \tilde{B} \in \mathcal{F}_{0}$ that satisfy (C1)-(C5), one has $\tilde{A}_{1} \leq A_{1}$ and $\tilde{B} \leq B$.

The construction of $A_{1}$ and $B$, based on a concatenation of the maximal dynamic $G$-clipper and the maximal dynamic $F$-regulator, is called the maximal dynamic $F$-regulator with delay $d$ and buffer $q$.

Proof: Suppose that $A_{1}$ and $B$ are as stated in the theorem. Theorem 4.1 then implies (C1), and also implies that $A_{1}(t) \leq$ $\left(A_{1} \star G\right)(t)$. Conditions (C4) and (C5) follow from Theorem 3.3(i) and (ii). To establish (C2), note that

$$
\begin{aligned}
A_{1}(t)-q & \leq\left(A_{1} \star G\right)(t)-q \\
& \leq \min _{0 \leq s \leq t}\left[A_{1}(s)+F^{*}(s, t)+q\right]-q \\
& =\left(A_{1} \star F^{*}\right)(t) \\
& =B(t) .
\end{aligned}
$$

Similarly, to establish (C3), note that

$$
\begin{aligned}
A_{1}(t) & \leq\left(A_{1} \star G\right)(t) \\
& \leq \min _{0 \leq s \leq t}\left\{A_{1}(s)+F^{*}(s, t+d)\right\} .
\end{aligned}
$$

Since $A_{1}(t) \leq A_{1}(s)$ for $s>t$ and $F^{*}$ is nonnegative, it, therefore, follows that $A_{1}(t) \leq\left(A_{1} \star F^{*}\right)(t+d)=B(t+d)$, which establishes (C3). Thus, (C1)-(C5) are satisfied as claimed.

Next, suppose that $\tilde{A}_{1}, \tilde{B} \in \mathcal{F}_{0}$ satisfy (C1)-(C5). From Theorem 3.3(iii), we know that, under the flow constraint in (C5) and the traffic constraint $(\mathrm{C} 4)$, we have

$$
\tilde{B} \leq \tilde{A}_{1} \star F^{*}
$$

Moreover, combining this with (C2) and (C3), we obtain

(C2') (Buffer constraint) $\tilde{A}_{1}(t)-q \leq\left(\tilde{A}_{1} \star F^{*}\right)(t)$ for all $t$.

(C3') (Delay constraint) $\tilde{A}_{1}(t) \leq\left(\tilde{A}_{1} \star F^{*}\right)(t+d)$ for all $t$.

The buffer constraint in (C2') can be rewritten as

$$
\tilde{A}_{1} \leq \tilde{A}_{1} \star F_{2}
$$


with $F_{2}(s, t)=F^{*}(s, t)+q$. Since $\tilde{A}_{1}(t) \in \mathcal{F}_{0}$ is nondecreasing in $t$ and $F^{*}(s, t)$ is nonnegative

$$
\tilde{A}_{1}(s)+F^{*}(s, t+d) \geq \tilde{A}_{1}(t), \quad s=t+1, \ldots, t+d .
$$

Due to the conditions in (27), the delay constraint in (C3') can be rewritten as

$$
\tilde{A}_{1} \leq \tilde{A}_{1} \star F_{3}
$$

with $F_{3}(s, t)=F^{*}(s, t+d)$. Using the idempotency and distributivity, the constraints in (26) and (28) are equivalent to

$\tilde{A}_{1}=\tilde{A}_{1} \oplus \tilde{A}_{1} \leq\left(\tilde{A}_{1} \star F_{2}\right) \oplus\left(\tilde{A}_{1} \star F_{3}\right)=\tilde{A}_{1} \star\left(F_{2} \oplus F_{3}\right)$.

Note that $\left(F_{2} \oplus F_{3}\right)(s, t)=G(s, t)$ for all $s<t$, where $G$ is defined in Theorem 5.1. Thus, $\tilde{A}_{1}$ conforms to the dynamic envelope $G$. Using Theorem 4.1(iii) and the assumption that $\tilde{A}_{1}$ satisfies (C1), it, therefore, follows that $\tilde{A}_{1}(t) \leq(\hat{A} \oplus G)^{*}(0, t)=$ $A_{1}(t)$. From the monotonicity of $\star$, we also have from (25) that

$$
\tilde{B} \leq \tilde{A}_{1} \star F^{*} \leq A_{1} \star F^{*}=B .
$$

We note that, for the special cases that $d=\infty$ (without delay constraint) and that $q=\infty$ (without buffer constraint), the results were previous obtained in [22, Ch. 9]. The result in Theorem 5.1 not only finds a representation of the optimal traffic regulator that satisfies both the delay and buffer constraints, but also provides a method for the implementation of such a regulator. In [19], the buffer and delay constraints are treated separately, and it is shown that the optimal solution can be implemented by the greedy flow controller, which discards packets only when needed. As shown in Theorem 5.1, the maximal dynamic $F$-regulator with delay $d$ and buffer $q$ is still the greedy flow controller as the maximal dynamic $G$-clipper discards packets only when needed.

Example 5.2(Work-Conserving Link with a Finite Buffer): In this example, we show that a work-conserving link with a finite buffer solves a traffic-regulation problem with a buffer constraint. Consider the work-conserving link with a time-varying capacity and a finite buffer in Example 4.2. As in Examples 3.4 and 4.2, let $A, A_{1}$, and $B$ be the input, effective input, and output of the link, respectively. As we have shown from Example 4.2, the effective input $A_{1}$ to the link is, in fact, the output of the maximal dynamic $G$-clipper with $G(s, t)=\hat{C}(s, t)+q$. Also, from Example 3.4, the output $B$ from the link is the output from the maximal dynamic $F$-regulator with $F(s, t)=\hat{C}(s, t)$. Thus, the link is a concatenation of the maximal dynamic $G$-clipper and the maximal dynamic $F$-regulator. We then have from Theorem 5.1 that the work-conserving link with a finite buffer $q$ is the maximal dynamic $F$-regulator with buffer $q$, where $F(s, t)=\hat{C}(s, t)$.

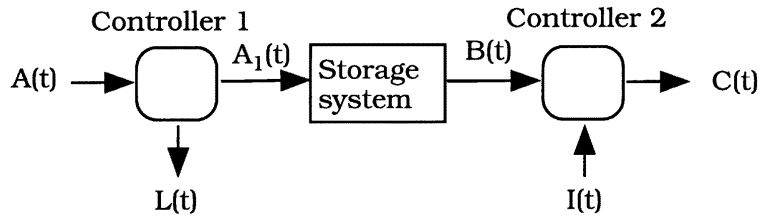

Fig. 1. Work-conserving link with a finite buffer.

There is a well-known duality interpretation for a work-conserving link with a finite buffer. One may view the cumulative capacity $C(t)$ as the cumulative number of tokens generated by time $t$. As in a leaky bucket, every packet needs to grab a token for its departure. Thus, packet losses occur when the buffer is full and token losses occur when the buffer is empty. To be precise, let $q(t)$ be the number of packets at the link at time $t, L(t)=A(t)-A_{1}(t)$ be the cumulative number of packet losses by time $t$, and $I(t)=C(t)-B(t)$ be the cumulative number of token losses by time $t$. Fig. 1 represents this system. One then has the following conditions of complementary slackness:

$$
\begin{aligned}
\mathbf{1}\{q(t)<q\}(L(t)-L(t-1)) & =0, & & \text { for all } t \\
\mathbf{1}\{q(t)>0\}(I(t)-I(t-1)) & =0, & & \text { for all } t
\end{aligned}
$$

where $1\{E\}=1$ if the event $E$ is true and 0 otherwise. As

$$
q(t)=A_{1}(t)-B(t)=(A(t)-C(t))+I(t)-L(t)
$$

the work-conserving link with a finite buffer solves the so-called Skorokhod reflection problem with two boundaries [28], where $A(t)-C(t)$ is the free process, $I(t)$ is the lower boundary process, and $L(t)$ is the upper boundary process (see, e.g., [18] and [19] for more detailed discussions of the reflection problem). Since the work-conserving link with a finite buffer also solves the buffer-constrained traffic-regulation problem, it follows from (24) that the upper boundary process of the reflection problem admits the following close-form representation (in terms of the free process):

$L(t)=\sup _{S} \sum_{i=1}^{m}\left[\left(A\left(t_{i}\right)-C\left(t_{i}\right)-\left(A\left(t_{i-1}\right)-C\left(t_{i-1}\right)\right)-q\right]^{+}\right.$

where $S=\left\{t_{0}, t_{1}, t_{2}, \ldots, t_{m}\right\}$ is any subset of $\{1,2, \ldots, t\}$ with $t_{0}=0<t_{1}<t_{2}<\cdots<t_{m}=t$. Using (2) and $B=A_{1} \star \hat{C}$, one can also show that the lower boundary process admits the following closed-form representation:

$I(t)=\sup _{S} \sum_{i=1}^{m-1} \max \left[\left(C\left(t_{i}\right)-A\left(t_{i}\right)-\left(C\left(t_{i-1}\right)-A\left(t_{i-1}\right)\right),-q\right]\right.$

where the sum in the right-hand side is 0 for $m=1$. We also note the queue-length process $q(t)$ can also be represented in closed form. Two representations based on min, max and plus operations were given in [14]. 
Example 5.3 (Multiple Leaky Buckets With Buffer and Delay Constraints): Now consider the maximal dynamic $F$-regulator with delay $d$ and buffer $q$ when

$$
F(s, s+t)=\min _{1 \leq i \leq K}\left[\rho_{i} t+\sigma_{i}\right], \quad t>0 .
$$

This corresponds to the case of multiple leaky buckets with the delay constraint $d$ and the buffer constraint $q$. In this case,

$$
\begin{aligned}
G(s, t) & =\min \left[\min _{1 \leq i \leq K}\left[\rho_{i}(t+d-s)+\sigma_{i}\right],\right. \\
& \left.\min _{1 \leq i \leq K}\left[\rho_{i}(t-s)+\sigma_{i}\right]+q\right] \\
= & \min _{1 \leq i \leq K}\left[\rho_{i}(t-s)+\sigma_{i}+\min \left[q, \rho_{i} d\right]\right] .
\end{aligned}
$$

Thus, one can construct the maximal dynamic $G$-clipper by feeding the input to $K$ parallel bufferless $\left(\sigma_{i}+\min \left[q, \rho_{i} d\right]\right.$, $\rho_{i}$ )-leaky buckets. A packet is discarded (or clipped) if it cannot be admitted to one of these $K$ leaky buckets. The output from the maximal dynamic $G$-clipper is then fed into another $K$ parallel $\left(\sigma_{i}, \rho_{i}\right)$-leaky buckets with buffer $q$. This example also shows that a direct calculation of the convolution in (12) may not be necessary, as leaky buckets are known to have recursive implementations.

To bound the cumulative loss for the maximal dynamic $G$-clipper in this example, we may apply the comparison result in Example 4.4. Consider $K$ maximal dynamic clippers, all subject to the same input. The $i$ th clipper is the maximal dynamic $G_{i}$-clipper with

$$
G_{i}(s, t)=\rho_{i}(t-s)+\sigma_{i}+\min \left[q, \rho_{i} d\right] .
$$

Let $L_{i}(t)$ be the cumulative number of losses by time $t$ at the $i$ th clipper. From Example 4.4, $\sum_{i=1}^{K} L_{i}(t)$ is an upper bound for the cumulative loss for the maximal dynamic $G$-clipper. Now $L_{i}(t)$ is much easier to compute, as it is simply the cumulative loss for a work-conserving link with capacity $\rho_{i}$ and buffer $\sigma_{i}+$ $\min \left[q, \rho_{i} d\right]$ in Example 5.2.

Example 5.4 (Bounding Losses by Segregation Between Buffer and Policer): We have shown in Theorem 5.1 that the maximal dynamic $F^{\prime}$-regulator with buffer $q$ is the optimal implementation to generate an output conforming to the dynamic envelope $F^{\prime}$ and subject to the buffer constraint $q$. In this example, we will show that segregation of buffer discard and policing discard provides an upper bound on the cumulative losses for the maximal dynamic $F^{\prime}$-regulator with buffer $q$.

As we have shown in Theorem 5.1, the first stage of the maximal dynamic $F^{\prime}$-regulator with buffer $q$ is the maximal dynamic $F$-clipper, where

$$
F(s, t)=F^{\prime}(s, t)+q .
$$

Let $A(t), D(t)$, and $L(t)$ be its input, output, and the cumulative losses by time $t$, i.e., $L(t)=A(t)-D(t)$. We now compare the cumulative losses $L(t)$ with the losses in another system made of two parts, as shown in Fig. 2. The first part is some causal system with storage capacity $q$. We know, however, that the first part discards packets as soon as the total backlogged packets in this system exceeds $q$. This operation is called buffer discard,

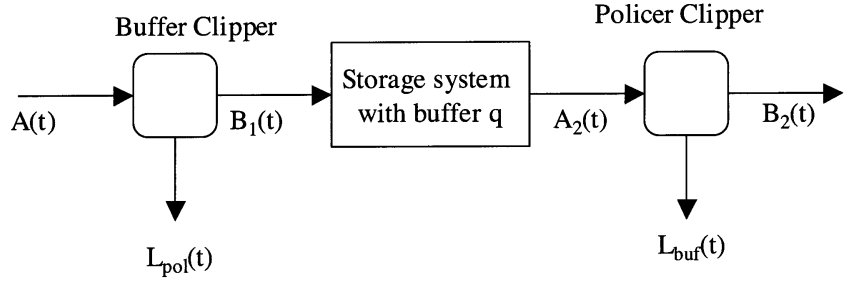

Fig. 2. Storage/policer system with separation between losses due to buffer discard and policing discard.

and the amount of buffer discarded packets by time $t$ is denoted by $L_{\mathrm{Buf}}(t)$. The second part is the maximal dynamic $F^{\prime}$-clipper referred to here as the policer. Packets are discarded as soon as the total output of the storage system exceeds the maximum output allowed by the policer. This operation is called policing discard, and the amount of discarded packets by time $t$ due to policing is denoted by $L_{\mathrm{Pol}}(t)$.

We show that $L(t) \leq L_{\mathrm{Buf}}(t)+L_{\mathrm{Pol}}(t)$. Let $B_{1}(t)$ be the output of the buffer clipper, $A_{2}(t)$ and $B_{2}(t)$ be the input and output of the policer clipper, respectively. As $B_{2}$ is the output of the maximal dynamic $F^{\prime}$-clipper

$$
B_{2}(t)-B_{2}(s) \leq F^{\prime}(s, t) .
$$

Now let $A_{1}$ be the effective input to the system, i.e.,

$$
A_{1}(t)=A(t)-L_{\mathrm{Buf}}(t)-L_{\mathrm{Pol}}(t) .
$$

Also, as shown in Fig. 2, we have

$$
L_{\mathrm{Buf}}(t)=A(t)-B_{1}(t)
$$

and

$$
L_{\mathrm{Pol}}(t)=A_{2}(t)-B_{2}(t) .
$$

Since $L_{\mathrm{Buf}}(t)+L_{\mathrm{Pol}}(t)$ is a nondecreasing function in $t$, we have from (32) that

$$
A_{1}(t)-A_{1}(s) \leq A(t)-A(s) .
$$

On the other hand, because the "storage system" is causal, it satisfies the flow constraint

$$
A_{2}(t) \leq B_{1}(t) .
$$

Since its storage space is limited to $q$, we also have

$$
B_{1}(t) \leq A_{2}(t)+q .
$$

Using (32) and (33), we have for all $0 \leq s<t$

$$
A_{1}(t)-A_{1}(s)=B_{1}(t)-B_{1}(s)-\left(L_{\mathrm{Pol}}(t)-L_{\mathrm{Pol}}(s)\right) .
$$

From (30), (31), (34), (36), and (37), it then follows that

$$
\begin{aligned}
A_{1}(t)-A_{1}(s) & \leq A_{2}(t)-A_{2}(s)-\left(L_{\mathrm{Pol}}(t)-L_{\mathrm{Pol}}(s)\right)+q \\
& =B_{2}(t)-B_{2}(s)+q \\
& \leq F^{\prime}(s, t)+q \\
& =F(s, t) .
\end{aligned}
$$

Combining (35) with (38), one notices that $A_{1}$ satisfies the same constraints as $D$. As $D$ is the output from the optimal implemen- 
tation in Theorem 5.1, it follows that $A_{1}(t) \leq D(t)$ or, equivalently, that $L(t) \leq L_{\mathrm{Buf}}(t)+L_{\mathrm{Pol}}(t)$.

Such a separation of resources between the "buffered system" and "policing system" is used in the estimation of loss probability for devising statistical CAC algorithms as proposed by Lo Presti et al. [26] (see also Elwalid et al. [17]).

\section{Dynamic Service Guarantees}

To guarantee end-to-end deterministic QoS for an input, the concept of service curves is developed in [1], [12], and [20] to work with the static envelopes of Cruz [10], [11]. A server is called a static $f$-server $\left(f \in \mathcal{F}_{0}\right)$ for an input sequence $A$ if its output sequence $B \equiv\{B(t), t=0,1,2, \ldots$,$\} satisfies$

$$
B(t) \geq \min _{0 \leq s \leq t}[A(s)+f(t-s)]
$$

for all $t$. Based on this, there is an associated filtering theory (under the ( $\mathrm{min},+$ ) algebra) in [1], [8], and [20] that eases design and computation of deterministic QoS. Our main objective in this section is to extend the concept of service curves and the associated filtering theory to the time-varying setting so that dynamic QoS can be guaranteed. The theory is based on the following definition of a dynamic $F$-server.

Definition 6.1 (Dynamic F-Server): A server is called a dynamic $F$-server $\left(F \in \tilde{\mathcal{F}}_{0}\right)$ for an input sequence $A$ if its output sequence satisfies $B \geq A \star F$, i.e.,

$$
B(t) \geq \min _{0 \leq s \leq t}[A(s)+F(s, t)]
$$

for all $t$. If the inequality in (40) is satisfied for all input sequences, then we say the dynamic $F$-server is universal. If the inequality in (40) is an equality, we say the dynamic $F$-server is exact.

Analogous to the filtering theory for static service curves, one may view the right-hand side of (40) as the output from a linear filter with the time-varying impulse response $F(s, t)$ under the $(\min ,+)$ algebra. If $F$ is a time-invariant bivariate function, then the dynamic $F$-server is equivalent to a static $f$-server, where $f(t)=F(0, t)$.

Clearly, the maximal dynamic $F$-regulator is a universal and exact dynamic $F^{*}$-server. Analogous to the time-invariant case, one has the following properties in Theorems $6.2-6.5$ for dynamic $F$-servers. The proofs are omitted, as they are identical to those in [8] and [9].

Theorem 6.2 (Concatenation): A concatenation of a dynamic $F_{1}$-server for an input sequence $A$ and a dynamic $F_{2}$-server for the output from the dynamic $F_{1}$-server is a dynamic $F$-server for $A$, where $F=F_{1} \star F_{2}$.

Theorem 6.3 (Filter Bank Summation): Consider an input sequence $A$. Let $B_{1}$ (respectively, $B_{2}$ ) be the output from a dynamic $F_{1}$-server (respectively, $F_{2}$-server) for $A$. The output from the "filter bank summation," denoted by $B$, is $B_{1} \oplus B_{2}$. The "filter bank summation" of a dynamic $F_{1}$-server for $A$ and a dynamic $F_{2}$-server for $A$ is then a dynamic $F$-server for $A$, where $F=F_{1} \oplus F_{2}$.

Theorem 6.4 (Feedback): Consider an input sequence $A \in$ $\mathcal{F}_{0}$ and a dynamic $F$-server for $B$, where $B=A \oplus A_{1}$, and $A_{1}$ is the output from the dynamic $F$-server. If $\inf _{t} F(t, t)>0$, then the feedback system is a dynamic $F^{*}$-server for $A$.

Theorem 6.5: Consider a dynamic $F_{2}$-server for $A$. Let $B$ be the output. Also, let $q=\sup _{t>0}[A(t)-B(t)]^{+}$be the maximum queue length at the server, where $x^{+}=\max (0, x)$. Let $d=$ $\inf \{\delta \geq 0: B(t+\delta) \geq A(t)$ for all $t\}$ be the maximum delay at the server. Suppose that $A$ conforms to the dynamic upper envelope $F_{1}$.

i) (Queue length) $q \leq \sup _{s} \sup _{t>s}\left[F_{1}^{*}(s, t)-F_{2}(s, t)\right]^{+}$.

ii) (Output burstiness) If $B \leq A$, then $B$ conforms to the dynamic upper envelope $F_{3}^{*}$, where

$$
F_{3}(s, t)=\max _{0 \leq \tau \leq s}\left[F_{1}^{*}(\tau, t)-F_{2}(\tau, s)\right]^{+} .
$$

iii) (Delay) $d \leq \inf \left\{\delta \geq 0: \sup _{s} \sup _{t \geq s}\left[F_{1}^{*}(s, t)-\right.\right.$ $\left.\left.F_{2}(s, t+\delta)\right] \leq 0\right\}$.

Remark 6.6: As the maximal dynamic $F$-regulator is a dynamic $F^{*}$-server, there is an intuitive explanation why the maximal dynamic $F$-regulator with delay $d$ and buffer $q$ is a concatenation of the maximal dynamic $G$-clipper (with $G$ being defined in Theorem 5.1) and the maximal dynamic $F$-regulator. As shown in Theorem 4.1, the output from the maximal dynamic $G$-clipper conforms to the dynamic envelope $G(s, t)=$ $\min \left[F^{*}(s, t+d), F^{*}(s, t)+q\right]$. When such an output is fed to the maximal dynamic $F$-regulator, one has from Theorem 6.5 that the delay at the maximal dynamic $F$-regulator is bounded above by $d$ and the queue length is also bounded above by $q$. Thus, both the delay constraint and buffer constraint are satisfied.

In the following, we illustrate the use of dynamic service guarantees by a dynamic window-flow-control problem.

Example 6.7 (Dynamic Window Flow Control): Consider a network with the input $A$ and the output $B$. Suppose that the network enforces a dynamic window flow control for the input $A$ with the dynamic window size $w(t)$. We assume that $\inf _{t} w(t)>0$. For the dynamic window-flow-control system, the effective input to the network, denoted by $A_{1}$, satisfies

$$
A_{1}(t)=\min [A(t), B(t)+w(t)]
$$

Observe that $B(t)+w(t)=(B \star H)(t)$, where $H$ is the function with $H(s, t)=\infty$ for $s<t$ and $H(t, t)=w(t)$. One may rewrite (41) as follows:

$$
A_{1}=A \oplus(B \star H) .
$$

Also, we assume that the network is a dynamic $F$-server for the effective input $A_{1}$, i.e.,

$$
B \geq A_{1} \star F .
$$

In conjunction with (42)

$B \geq A_{1} \star F=(A \oplus(B \star H)) \star F=(A \star F) \oplus(B \star(H \star F))$

where we apply the distributive property and the associativity of $\star$. Since we assume that $\inf _{t} w(t)>0$

$$
\inf _{t}(H \star F)(t, t)=\inf _{t}[H(t, t)+F(t, t)] \geq \inf _{t} w(t)>0 .
$$


We then have from Lemma 2.2(iii) that

$$
B \geq A \star F \star(H \star F)^{*} .
$$

Thus, the dynamic window-flow-control system is a dynamic $F \star(H \star F)^{*}$-server.

\section{DYNAMIC SCED SchEDULING ALGORITHM}

In this section, we define a scheduling algorithm, called the dynamic SCED algorithm, which we will show achieves the dynamic service guarantees in Section VI.

Consider a server with a time-varying capacity. Let $c(t)$ be the maximum number of packets that can be served at time $t$, and $\hat{C}(s, t)=\sum_{\tau=s+1}^{t} c(\tau)$ be the cumulative capacity in the interval $[s+1, t]$. A policy is called the EDF if the server schedules the packets according to their deadlines. Note that the EDF policy is work conserving, i.e., the server serves packets whenever there are packets at the server.

Now consider feeding $n$ streams of inputs to such a server. Let $A_{i}(t)$ be the cumulative number of packet arrivals of the $i$ th stream up to time $t$. Each packet is assigned a deadline. We assume that the deadlines within the same stream are nondecreasing. Also, let $N_{i}(t)$ be the number of packets from the $i$ th stream that have deadlines not greater than $t$. As we assume the deadlines for each stream is nondecreasing, packet $k$ from stream $i$ is assigned the deadline $D_{i, k}$ from the following inverse mapping:

$$
D_{i, k}=\inf \left\{t: t \geq 0 \text { and } N_{i}(t) \geq k\right\} .
$$

Theorem 7.1: Suppose that the server is operated under the EDF policy. The necessary and sufficient condition for every packet to be served not later than its deadline is

$$
\sum_{i \in S} N_{i}(t) \leq \min _{0 \leq s \leq t}\left[\sum_{i \in S} A_{i}(s)+\hat{C}(s, t)\right]
$$

for all $t$ and for every $S$ that is a subset of $\{1,2, \ldots, n\}$.

Proof: i) We first prove the necessary part. Let $B(t)$ be the cumulative number of packet departures from all streams up to time $t$. Since the EDF policy is work conserving, we have from Example 3.4 that

$$
B(t)=\min _{0 \leq s \leq t}\left[\sum_{i=1}^{n} A_{i}(s)+\hat{C}(s, t)\right] .
$$

As we assume that every packet is served not later than its deadline

$$
\sum_{i=1}^{n} N_{i}(t) \leq B(t)=\min _{0 \leq s \leq t}\left[\sum_{i=1}^{n} A_{i}(s)+\hat{C}(s, t)\right]
$$

for all $t$.

Let $S$ be a subset of $\{1,2, \ldots, n\}$. Suppose that we now only have the streams in $S$ (the packets from other streams are discarded). Based on a standard sample path argument, it is clear that, under the EDF policy, every packet is still served not later than its deadline. Following the same argument as in (46) yields (45). ii) We prove the sufficient part by contradiction, as in [25]. Suppose that the first packet that misses its deadline occurs at time $t$. Let $\tau^{*}$ be the last slot no later than $t$ such that the server serves less than $c\left(\tau^{*}\right)$ packets. Since the EDF policy is work conserving, $\tau^{*}<t$, as there is at least one stream $i$ packet backlogged at time $t$. Moreover, there are exactly $\hat{C}\left(\tau^{*}, t\right)$ packets served in the interval $\left[\tau^{*}+1, t\right]$.

Now let $s^{*}$ be the last slot in the interval $\left[\tau^{*}+1, t\right]$, during which a packet with deadline greater than $t$ is served. If all the packets served during the interval $\left[\tau^{*}+1, t\right]$ have deadlines less than or equal to $t$, then define $s^{*}=\tau^{*}$ (in this case, there are no backlogged packets at the end of slot $s^{*}$ ). Thus, during the interval $\left[s^{*}+1, t\right]$, exactly $\hat{C}\left(s^{*}, t\right)$ packets are served, and each of these packets has a deadline that is less than or equal to $t$.

Let $S$ be the set of streams that are not backlogged at the end of slot $s^{*}$. We claim that those packets served in $\left[s^{*}+1, t\right]$ can only come from the streams in $S$. Suppose that stream $i$ is not in $S$. Since there is a packet with deadline greater than $t$ that is served in slot $s^{*}$, all the backlogged stream $i$ packets at the end of slot $s^{*}$ must have deadlines greater than $t$. This implies all the stream $i$ packets with deadlines not greater than $t$ have been served, as we assume the deadlines are nondecreasing within the same stream. Thus, those packets served in $\left[s^{*}+1, t\right]$ can only come from the streams in $S$, as those packets have deadlines less than or equal to $t$.

Now suppose that stream $i$ is in $S$. As there are no backlogged stream $i$ packets at the end of slot $s^{*}$, all the stream $i$ packets that arrive not later than $s^{*}$ have been served. Thus, the number of stream $i$ packets that can be served in $\left[s^{*}+1, t\right]$ is bounded above by $\left(N_{i}(t)-A_{i}\left(s^{*}\right)\right)^{+}$. This, in turn, implies that the number of packets served in $\left[s^{*}+1, t\right]$ is bounded above by $\sum_{i \in S}\left(N_{i}(t)-A_{i}\left(s^{*}\right)\right)^{+}$. As there is a packet that misses its deadline at time $t$, the bound is strict. Thus,

$$
\hat{C}\left(s^{*}, t\right)<\sum_{i \in S}\left(N_{i}(t)-A_{i}\left(s^{*}\right)\right)^{+}=\sum_{i \in S^{\prime}}\left[N_{i}(t)-A_{i}\left(s^{*}\right)\right]
$$

for some $S^{\prime}$ that is a subset of $S$ with $N_{i}(t) \geq A_{i}\left(s^{*}\right)$. As $S^{\prime}$ is a subset of $\{1,2, \ldots, n\}$, we have a contradiction to (45).

Lemma 7.2: Suppose we choose $N_{i}=A_{i} \star F_{i}$ some $F_{i} \in$ $\tilde{\mathcal{F}}_{0}, i=1, \ldots, n$. If $\sum_{i=1}^{n} F_{i}(s, t) \leq \hat{C}(s, t)$ for all $0 \leq s \leq$ $t$, then all the packets are served not later than their deadlines.

Such a deadline assignment scheme is called the dynamic SCED algorithm in this paper.

Proof: It suffices to verify that the sufficient condition in Theorem 7.1 is satisfied. Note that for every $S$ in $\{1,2, \ldots, n\}$

$$
\begin{aligned}
\sum_{i \in S} N_{i}(t) & =\sum_{i \in S} \min _{0 \leq s \leq t}\left[A_{i}(s)+F_{i}(s, t)\right] \\
& \leq \min _{0 \leq s \leq t} \sum_{i \in S}\left[A_{i}(s)+F_{i}(s, t)\right] \\
& =\min _{0 \leq s \leq t}\left[\sum_{i \in S} A_{i}(s)+\sum_{i \in S} F_{i}(s, t)\right] \\
& \leq \min _{0 \leq s \leq t}\left[\sum_{i \in S} A_{i}(s)+\sum_{i=1}^{n} F_{i}(s, t)\right] \\
& \leq \min _{0 \leq s \leq t}\left[\sum_{i \in S} A_{i}(s)+\hat{C}(s, t)\right]
\end{aligned}
$$


where we use $F_{i}(s, t) \geq 0$ and $\sum_{i=1} F_{i}(s, t) \leq \hat{C}(s, t)$ in the last two inequalities.

The next lemma implies that deadlines in the dynamic SCED algorithm can be assigned in real time. Specifically, if packet $k$ from stream $i$ arrives during slot $t, D_{i, k}$ can be computed without knowledge of $A_{i}(s)$ for $s>t$.

Lemma 7.3: Suppose packet $k$ from stream $i$ arrives during slot $t$. Under the dynamic SCED algorithm, $D_{i, k}=D_{i, k}(t)$ where

$$
\begin{aligned}
& D_{i, k}(t) \\
& \quad=\inf \left\{\Delta: \Delta \geq t \text { and } \min _{0 \leq u \leq t-1}\left[A_{i}(u)+F_{i}(u, \Delta)\right] \geq k\right\} .
\end{aligned}
$$

Proof: Note that, under the dynamic SCED algorithm

$$
D_{i, k}=\inf \left\{\Delta: \Delta \geq 0 \text { and }\left(A_{i} \star F_{i}\right)(\Delta) \geq k\right\} .
$$

Since packet $k$ arrives at time $t$, we have $A_{i}(u)<k$ for $u<t$. Thus, $\left(A_{i} \star F_{i}\right)(\Delta) \leq A_{i}(\Delta)<k$ when $\Delta \leq t-1$, which implies that $D_{i, k} \geq t$ by definition of $D_{i, k}$ in (48). Therefore, by definition of $D_{i, k}$, we have

$$
\begin{aligned}
k & \leq\left(A_{i} \star F_{i}\right)\left(D_{i, k}\right) \\
& \leq \min _{0 \leq u \leq t-1}\left[A_{i}(u)+F_{i}\left(u, D_{i, k}\right)\right] .
\end{aligned}
$$

By definition of $D_{i, k}(t)$, this implies $D_{i, k}(t) \leq D_{i, k}$. To show the reverse inequality, note that by definition of $D_{i, k}(t)$, we have

$$
\min _{0 \leq u \leq t-1}\left[A_{i}(u)+F_{i}\left(u, D_{i, k}(t)\right)\right] \geq k .
$$

Since $A_{i}(u) \geq k$ for $u \geq t$ and $F_{i}$ is nonnegative, inequality (49) implies that $\left(A_{i} \star F_{i}\right)\left(D_{i, k}(t)\right) \geq k$. By definition of $D_{i, k}$, this then implies that $D_{i, k} \leq D_{i, k}(t)$.

In Theorem 7.4, we state the admission criteria for the dynamic SCED algorithm for a server with a time-varying capacity. Once the admission criteria are met, the dynamic SCED algorithm can then be used for providing dynamic service guarantees.

Theorem 7.4: A set of $n$ arrival streams, indexed $i=1, \ldots, n$, arrives to a server. The arrival sequence of the $i$ th stream is denoted by $A_{i}$, and is known to conform to the dynamic upper envelope $G_{i}$. The server has a time-varying capacity to serve up to $c(t)$ packets during slot $t$. Under the dynamic SCED algorithm, the server is a dynamic $F_{i}$-server for $A_{i}$ for all $i=1, \ldots, n$ if the following condition is satisfied for all $s \leq t$ :

$$
\sum_{i=1}^{n}\left(G_{i} \star F_{i}\right)(s, t) \leq \hat{C}(s, t) .
$$

Proof: As we assume that $A_{i}$ conforms to the dynamic upper envelope $G_{i}$, we have from Lemma 3.2(ii) that $A_{i}=A_{i}$ ᄎ $G_{i}$. Thus,

$$
N_{i}=A_{i} \star F_{i}=\left(A_{i} \star G_{i}\right) \star F_{i}=A_{i} \star\left(G_{i} \star F_{i}\right)
$$

where we apply the associativity of $\star$. From Lemma 7.2, it then follows that all the packets are served before their deadlines. Denote by $B_{i}(t)$ the cumulative number of departures from stream $i$ by time $t$. Thus,

$$
B_{i} \geq N_{i}=A_{i} \star F_{i}
$$

and the server is a dynamic $F_{i}$-server for $A_{i}$ for all $i=1, \ldots, n$.

\section{CONCLUSIONS}

By extending the filtering theory under the $(\mathrm{min},+)$ algebra to the time-varying setting, we solved the problem of constrained traffic regulation. For a constrained traffic-regulation problem with maximum tolerable delay $d$ and maximum buffer size $q$, we showed that the optimal regulator that generates the output traffic conforming to a dynamic envelope $F$ and minimizes the number of discarded packets is a concatenation of the maximal dynamic $G$-clipper with $G(s, t)=\min \left[F^{*}(s, t+d), F^{*}(s, t)+q\right]$ and the maximal dynamic $F$-regulator. To provide dynamic service guarantees in a network, we developed the concept of the dynamic $F$-server as a basic network element. We showed that dynamic servers can be joined by concatenation, "filter bank summation," and feedback to form a composite dynamic server. We also proposed the dynamic SCED scheduling algorithm to achieve dynamic service guarantees for a work-conserving link subject to multiple inputs.

One possible application of the time-varying filtering theory is dynamic admission control. For a given connection $i$, we may define a service curve $f_{i}$ to be guaranteed over the interval $\left[a_{i}+\right.$ $\left.1, b_{i}\right]$ if a dynamic service curve $F_{i}$ is guaranteed, where

$$
F_{i}(s, t)=\left\{\begin{array}{l}
0, \quad \text { if } s \leq a_{i} \text { and } t \leq a_{i} \\
f_{i}\left(t-a_{i}\right), \quad \text { if } s \leq a_{i} \text { and } a_{i} \leq t \leq b_{i} \\
f_{i}(t-s), \quad \text { if } a_{i} \leq s \leq b_{i} \text { and } a_{i} \leq t \leq b_{i} \\
f_{i}\left(b_{i}-s\right), \quad \text { if } a_{i} \leq s \leq b_{i} \text { and } t>b_{i} \\
0, \quad \text { if } s \geq b_{i} \text { and } t \geq b_{i} \\
f_{i}\left(b_{i}-a_{i}\right), \quad \text { if } s<a_{i} \text { and } t>b_{i} .
\end{array}\right.
$$

For such a definition for dynamic service guarantees, an interesting problem is to find the relaxation time $r_{i}$ such that connection $i$ has virtually no impact on the admission criteria in Theorem 7.4 after $b_{i}+r_{i}$.

Finally, we note that our approach is also applicable in the continuous-time setting, as shown in [21] and [23]. We also note that the bivariate function $F$ could be random. By specifying the probabilistic characteristics of the bivariate function $F$, it is possible to provide probabilistic guarantees. Previous results along this line could be found in [7] and [13].

\section{REFERENCES}

[1] R. Agrawal, R. L. Cruz, C. Okino, and R. Rajan, "Performance bounds for flow control protocols," IEEE Trans. Networking, vol. 7, pp. 310-323, June 1999.

[2] R. Agrawal and R. Rajan, "A general framework for analyzing schedulers and regulators in integrated services network," in Proc. 34th Annu. Allerton Commun., Contr, and Computing Conf., Oct. 1996, pp. 239-248.

[3] - " "Open and closed loop control in integrated services networks," in Proc. 36th IEEE CDC, vol. 2, Dec. 1997, pp. 1798-1803. 
[4] R. Agrawal, F. Baccelli, and R. Rajan, "An algebra for queueing networks with time varying service and its application to the analysis of integrated service networks," Elect. Comput. Eng. Dept., Univ. Wisconsin-Madison, Madison, WI, Tech. Rep. ECE-98-2. [Online]. Available: http://www.ece.wisc.edu/ agrawal, May 1998.

[5] F. Baccelli, G. Cohen, G. Oslder, and J. Quadrat, Synchronization and Linearity: An Algebra for Discrete Event Systems. New York: Wiley, 1992.

[6] C. S. Chang, "Stability, queue length, and delay of deterministic and stochastic queueing networks," IEEE Trans. Automat. Contr., vol. 39, pp. 913-931, May 1994.

[7] - "On the exponentiality of stochastic linear systems under the max-plus algebra," IEEE Trans. Automat. Contr., vol. 41, pp. 1182-1188, Aug. 1996.

[8] — "On deterministic traffic regulation and service guarantees: A systematic approach by filtering," IEEE Trans. Inform. Theory, vol. 44, pp. 1097-1110, May 1998.

[9] - "Matrix extensions of the filtering theory for deterministic traffic regulation and service guarantees," IEEE J. Select. Areas Commun., vol. 16, pp. 708-718, June 1998.

[10] R. L. Cruz, "A calculus for network delay, Part I: Network elements in isolation," IEEE Trans. Inform. Theory, vol. 37, pp. 114-131, Jan. 1991.

[11] — "A calculus for network delay, Part II: Network analysis," IEEE Trans. Inform. Theory, vol. 37, pp. 132-141, Jan. 1991.

[12] — , "Quality of service guarantees in virtual circuit switched networks," IEEE J. Select. Areas Commun., vol. 13, pp. 1048-1056, Aug. 1995.

[13] - "Quality of service management in integrated services networks," in Proc. 1st Semiannu. Res. Rev., La Jolla, CA, June 1996.

[14] R. L. Cruz and H.-N. Liu, "Single server queues with loss: A formulation," in Proc. CISS Baltimore, MD, Mar. 1993.

[15] R. L. Cruz and C. M. Okino, "Service guarantees for a flow control," presented at the 34th Allerton Comm., Cont., and Comp. Conf., Monticello, IL, Oct. 1996, Preprint, first version.

[16] R. L. Cruz and M. Taneja, "An analysis of traffic clipping," in Proc. Inform. Sci., and Syst. Conf. Princeton, NJ, 1998.

[17] A. Elwalid, D. Mitra, and R. Wenworth, "A new approach for allocating buffers and bandwidth to heterogeneous, regulated traffic in ATM node," IEEE J. Select. Areas Commun., vol. 13, pp. 1048-1056, Aug. 1995.

[18] J. M. Harrison, Brownian Motion and Stochastic Flow Systems. New York: Wiley, 1985.

[19] T. Konstantopoulos and V. Anantharam, "Optimal flow control schemes that regulate the burstiness of traffic," IEEE/ACM Trans. Networking, vol. 3, pp. 423-432, Aug. 1995.

[20] J. Y. Le Boudec, "Application of network calculus to guaranteed service networks," IEEE Trans. Inform. Theory, vol. 44, pp. 1087-1096, May 1998.

[21] J. Y. Le Boudec and P. Thiran, "A note on time and space methods in network calculus," in Proc. Int. Broadband Commun. Seminar, Zurich, Switzerland, Feb. 1998, pp. 267-272.

[22] —-, Network Calculus: A Theory of Determinstic Queueing Systems for the Internet. Berlin, Germany: Springer-Verlag, 2001.

[23] — - "Network calculus using min-plus system theory," in High Performance Networks for Multimedia Applications, A. Danthine, O. Spaniol, W. Effelsberg, and D. Ferrari, Eds. Norwell, MA: Kluwer, 1999.

[24] J. Y. Le Boudec and A. Ziedins, "A CAC algorithm for VBR connections over a VBR trunk," in Proc. ITC 15, Washington, DC, June 1997, pp. $59-70$.

[25] C. L Liu and J. W. Layland, "Scheduling algorithms for multiprogramming in a hard-real-time environment," J. Assoc. Comput. Mach., vol. 20, pp. 46-61, 1973.

[26] F. Lo Presti, Z.-L. Zhang, Z.-L. D, Z.-L. Towsley, and J. Kurose, "Source time scale and optimal buffer/bandwidth trade-off for regulated traffic in an ATM node," in Proc. IEEE Infocom'97, Kobe, Japan, pp. 675-682.

[27] H. Sariowan, R. L. Cruz, and G. C. Polyzos, "SCED: A generalized scheduling policy for guaranteeing quality-of-service," IEEE/ACM Trans. Networking, vol. 7, pp. 669-684, Oct. 1999.

[28] A. Skorokhod, "Stochastic equations for diffusion processes in a bounded region," Theory Probab. Appl., vol. 6, pp. 264-274, 1961.

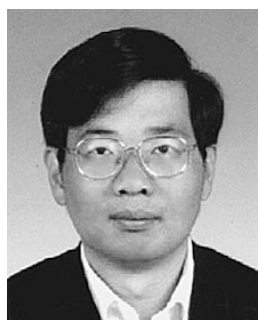

Cheng-Shang Chang (S'85-M'86-SM'93) received the B.S. degree from the National Taiwan University, Taipei, Taiwan, R.O.C., in 1983, and the M.S. and Ph.D. degrees from Columbia University, New York, NY, in 1986 and 1989, respectively, all in electrical engineering.

From 1989 to 1993, he was a Research Staff Member with the IBM Thomas J. Watson Research Center, Yorktown Heights, NY. Since 1993, he has been with the Department of Electrical Engineering, National Tsing Hua University, Taiwan, R.O.C., where he is a Professor. His current research interests are concerned with queueing theory, stochastic scheduling, and performance evaluation of telecommunication networks and parallel processing systems. He authored Performance Guarantees in Communication Networks (New York: Springer, 2000) and served as an Editor for Operations Research from 1992 to 1999.

Dr. Chang was the recipient of an IBM Outstanding Innovation Award in 1992, an IBM Faculty Partnership Award in 2001, and Outstanding Research Awards from the National Science Council, Taiwan, R,O.C., in 1999 and 2001, respectively.

Rene L. Cruz (S'80-M'84-SM'90) received the B.S. and Ph.D. degrees from the University of Illinois at Urbana-Champaign, in 1980 and 1987, respectively, and the S.M. degree from the Massachusetts Institute of Technology (MIT), Cambridge, in 1982, all in electrical engineering.

Since 1987, he has been with the University of California at San Diego, La Jolla, where he is currently a Professor of electrical and computer engineering.

Dr. Cruz was a program co-chair of the 2001 IEEE INFOCOM Conference and a general co-chair of the 20001 Association for Computing Machinery (ACM) SIGCOMM Conference. He was an associate editor for the IEEE TRANSACTIONS ON INFORMATION THEORY from 1994 to 1997.

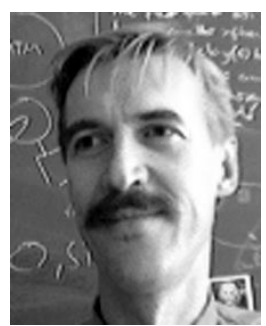

Jean-Yves Le Boudec graduated from the Ecole Normale Superieure de Saint-Cloud, Paris, France. He received the Doctorate degree from the University of Rennes, Rennes, France, in 1984.

In 1987, he joined Bell Northern Research, Ottawa, ON, Canada, where he was a Member of Scientific Staff with the Network and Product Traffic Design Department. In 1988, he joined the IBM Zurich Research Laboratory, Zurich, Switzerland, where he was Manager of the Customer Premises Network Department. In 1994, he joined the professor at Ecole Polytechnic Fédérale de Lausanne (EPFL). Lausanne, Switzerland, as a Professor and is currently a Full Professor. His interests are in the architecture and performance of communication systems.

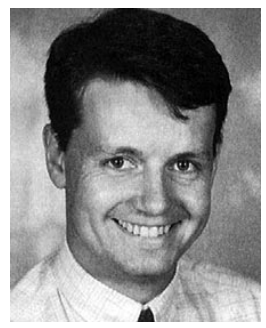

Patrick Thiran (S'88-M'90) received the Electrical Engineering Degree from the Université Catholique de Louvain, Louvain-la-Neuve, Belgium, in 1989, the M.S. degree in electrical engineering from the University of California at Berkeley, in 1990, and the $\mathrm{Ph} . D$. degree from the Ecole Polytechnic Fédérale de Lausanne (EPFL), Lausanne, Switzerland, in 1996.

While with the EPFL, he was initially with the Circuits and Systems Group, where he was involved with nonlinear circuits and systems (including neural networks) until 1996. He then joined the Communication Networks Laboratory, EPFL, where he became a Professor (Professeur titulaire) in 1998. From 2000 to 2001, he was with Sprintlabs, Burlingame, CA. He is currently an Assistant Professor with the School of Computer and Communication Sciences, EPFL. His research interests are communication networks, performance analysis, dynamical systems, and stochastic models.

Dr. Thiran is a Fellow of the Belgian American Educational Foundation. He was an associate editor for the IEEE TRANSACTIONS ON CIRCUITS AND SYSTEMS from 1997 to 1999. He was the recipient of the 1996 EPFL Doctoral Prize. 